\title{
Author Correction: Old news on quasar viscosity
}

\section{Robert Antonucci}

Correction to: Nature Astronomy https://doi.org/10.1038/s41550-018-0521-1, published online 3 July 2018.

In the version of this Correspondence originally published, the wrong paper was given for ref. 1; instead of 'Shields, G. A. Nature 273, 519-520 (1978)' it should have been 'Shields, G. A. Nature 272, 706-708 (1978)'. This has now been corrected. 